\title{
Evolutionary history, immigration history, and the extent of diversification in community assembly
}

\author{
Matthew L. Knope ${ }^{1}$, Samantha E. Forde ${ }^{2}$ and Tadashi Fukami ${ }^{1 *}$ \\ ${ }^{1}$ Department of Biology, Stanford University, Stanford, CA, USA \\ ${ }^{2}$ Department of Ecology and Evolutionary Biology, University of California Santa Cruz, Santa Cruz, CA, USA
}

\section{Edited by:}

Diana Reid Nemergut, University of Colorado Boulder, USA

\section{Reviewed by:}

Susannah Green Tringe, DOE Joint Genome Institute, USA

Uli Stingl, King Abdullah University of Science and Technology KAUST,

Saudi Arabia

\section{*Correspondence:}

Tadashi Fukami, Department of Biology, Stanford University, 371 Serra Mall, Stanford, CA 94305-5020, USA.

e-mail: fukamit@stanford.edu
During community assembly, species may accumulate not only by immigration, but also by in situ diversification. Diversification has intrigued biologists because its extent varies even among closely related lineages under similar ecological conditions. Recent research has suggested that some of this puzzling variation may be caused by stochastic differences in the history of immigration (relative timing and order of immigration by founding populations), indicating that immigration and diversification may affect community assembly interactively. However, the conditions under which immigration history affects diversification remain unclear. Here we propose the hypothesis that whether or not immigration history influences the extent of diversification depends on the founding populations' prior evolutionary history, using evidence from a bacterial experiment. To create genotypes with different evolutionary histories, replicate populations of Pseudomonas fluorescens were allowed to adapt to a novel environment for a short or long period of time (approximately 10 or 100 bacterial generations) with or without exploiters (viral parasites). Each evolved genotype was then introduced to a new habitat either before or after a standard competitor genotype. Most genotypes diversified to a greater extent when introduced before, rather than after, the competitor. However, introduction order did not affect the extent of diversification when the evolved genotype had previously adapted to the environment for a long period of time without exploiters. Diversification of these populations was low regardless of introduction order. These results suggest that the importance of immigration history in diversification can be predicted by the immigrants' evolutionary past. The hypothesis proposed here may be generally applicable in both micro- and macro-organisms.

Keywords: adaptive peaks, colonization, dispersal, eco-evolutionary dynamics, fitness trade-off, historical contingency, niche pre-emption, priority effect

\section{INTRODUCTION}

During community assembly, species can accumulate both ecologically, by immigration, and evolutionarily, by in situ diversification. Traditionally, these processes have been studied separately by ecologists on one hand and evolutionary biologists on the other. However, an increasing number of recent studies on both microand macro-organisms consider immigration and diversification simultaneously (e.g., Hubbell, 2001; Gillespie, 2004; Fukami et al., 2007). Much of this change has been prompted by the growing appreciation of "eco-evolutionary dynamics," where ecological and evolutionary processes operate interactively at the same time scales (Hairston et al., 2005; Schoener, 2011). One idea that is emerging from eco-evolutionary research is that the extent of diversification can be affected by stochastic differences in immigration history, such as the relative timing and order of immigration of competing genotypes (Silvertown, 2004; Fukami et al., 2007; Gillespie and Emerson, 2007; Seehausen, 2007). This interactive effect of immigration and diversification arises because early arriving immigrants pre-empt available niches, thereby suppressing diversification of late-arriving immigrants - an evolutionary "priority effect” (Samuels and Drake, 1997; Chase, 2003; Gillespie, 2004;
Silvertown, 2004; Fukami et al., 2007; Gillespie and Emerson, 2007; Seehausen, 2007; Urban and De Meester, 2009). It remains unclear, however, under what conditions immigration history influences the extent of diversification. As a first step toward answering this question, this paper proposes one hypothesis, namely that the importance of immigration history is determined by the degree of prior adaptation of founding populations to the new environment. To provide empirical support for this hypothesis, we present the results of an experiment that involved the plant-colonizing bacterium Pseudomonas fluorescens and its viral parasite, phage $\Phi 2$.

Pseudomonas fluorescens is a useful model system to study diversification. It has been shown to rapidly diversify into spatial niche specialists by de novo mutation when propagated in a novel habitat (static vial containing nutrient-rich liquid medium; Rainey and Travisano, 1998). Primary classes of niche specialists that emerge through this adaptive radiation include "smooth morphs" (SM), which resemble the ancestral type and primarily colonize the liquid phase, "wrinkly spreader (WS) morphs," which form a biofilm at the air-liquid interface, and "fuzzy spreader (FS) morphs," which appear to inhabit the bottom of the vial (Rainey and Travisano, 1998). Heritable variation exists within each class, and evolved 
morphotypes coexist via negative frequency-dependent selection (Rainey and Travisano, 1998; Fukami et al., 2007; Meyer and Kassen, 2007). Because reproduction in P. fluorescens is entirely asexual, genotypes are analogous to species in other organisms (Kassen et al., 2004). Further, previous work has shown strong effects of the presence of phage $\Phi 2$ on $P$. fluorescens diversification via reduction in bacterial density and selection for phage resistance (Buckling and Rainey, 2002).

Using $P$. fluorescens and phage $\Phi 2$, we examined the effects of two aspects of prior evolutionary history on the role of immigration history in diversification: how long immigrants have previously evolved in an environment similar to the new habitat, and whether or not immigrants have previously evolved in the presence of the exploiters that they encounter in the new habitat. We examined these two aspects because both theory (Wright, 1932; Dobzhansky, 1937; Simpson, 1953; Kauffman, 1989; Whitlock et al., 1995; Schluter, 2000) and observations from the field (Simpson, 1953; Schluter, 2000) suggest that the degree of similarity between the prior environment that immigrants come from and the new environment (both biotic and abiotic) is a key factor affecting diversification in the new environment. In our experiment, we allowed replicate bacterial populations to evolve in a novel environment, with and without phage $\Phi 2$, for approximately 10 or 100 generations. We then introduced each of the evolved genotypes into a new habitat either before or after a standard competitor genotype to evaluate the effect of immigration history. We found that whether or not the extent of diversification was influenced by immigration history did depend significantly on the adaptation history of the immigrants prior to immigration. We will discuss possible mechanisms for this effect, and suggest that they may be generally applicable to other systems of both microand macro-organisms, with the caveat that results of microbial experiments should not be extrapolated uncritically (Carpenter, 1996; Cadotte et al., 2005).

\section{MATERIALS AND METHODS CREATION OF GENOTYPES WITH DIFFERENT PRIOR EVOLUTIONARY HISTORIES}

We propagated six independent replicate lineages of wild type P. fluorescens SBW25 (Rainey and Travisano, 1998) at $28^{\circ} \mathrm{C}$ in static 25-ml universal vials with loose caps containing $6 \mathrm{ml}$ of standard King's B (KB) liquid medium (Rainey and Travisano, 1998; Fukami et al., 2007; Meyer and Kassen, 2007), in the absence of phage (three lineages) and in the presence of phage (three lineages). All of these lineages were initiated with a common isogenic SM population of $P$. fluorescens. Approximately $3 \times 10^{5}$ particles of phage SBW25 2 were also introduced to all appropriate vials at the start of the propagation. Subsequently, $60 \mu \mathrm{l}$ of culture was transferred to fresh media every $48 \mathrm{~h}$ for 30 days. We harvested $1 \mathrm{ml}$ of each replicate at every transfer, starting on day 1 . Immediately after each harvest, the samples were stored in $70 \%$ glycerol at $-80^{\circ} \mathrm{C}$. From this collection of evolved SM genotypes, we chose genotypes isolated after 3 days (corresponding to approximately 10 bacterial generations) and 27 days (approximately 100 bacterial generations) of propagation to represent brief and long periods of prior adaptation to the novel environment, respectively. Thus we had four treatments of prior evolutionary history: short or long periods of adaptation, each in either the presence or absence of exploiters. It has been shown under laboratory conditions that $P$. fluorescens evolves to have increased general resistance to phage over time when continuously exposed to phage (Brockhurst et al., 2003).

\section{MANIPULATION OF PRIOR EVOLUTIONARY HISTORY AND IMMIGRATION HISTORY}

Using the same conditions as above, we inoculated fresh vials with an isogenic population of SM genotype isolated from an evolved lineage (one of each of the three replicates for all four of the varied evolutionary histories as described above; hereafter referred to as focal genotype) and that of a common ancestral SM genotype with a neutral lacZ genetic marker (Zhang and Rainey, 2007; used as a standard competitor; hereafter referred to as competitor genotype). Use of a lac $Z$ lineage as the competitor genotype allowed the origin of each bacterial cell to be determined by observation of colonies developed on KB agar plates supplemented with X-gal (see below).

The following 12 treatments of immigration history were used: (1) focal genotype with 3-day prior history with phage (hereafter called 3W) introduced by itself; (2) focal genotype with 3-day prior history without phage (3WO) introduced by itself; (3) focal genotype with 27 -day prior history with phage $(27 \mathrm{~W})$ introduced by itself; (4) focal genotype with 27-day prior history without phage (27WO) introduced by itself; (5) competitor genotype introduced first, then $3 \mathrm{~W}$ introduced $24 \mathrm{~h}$ later; (6) $3 \mathrm{~W}$ introduced first, then competitor genotype introduced $24 \mathrm{~h}$ later; (7) competitor genotype introduced first, then $3 \mathrm{WO}$ introduced $24 \mathrm{~h}$ later; (8) 3WO introduced first, then competitor genotype introduced $24 \mathrm{~h}$ later; (9) competitor genotype introduced first, then $27 \mathrm{~W}$ introduced $24 \mathrm{~h}$ later; (10) $27 \mathrm{~W}$ introduced first, then competitor genotype introduced $24 \mathrm{~h}$ later; (11) competitor genotype introduced first, then $27 \mathrm{WO}$ introduced $24 \mathrm{~h}$ later; and (12) $27 \mathrm{WO}$ introduced first, then competitor genotype introduced $24 \mathrm{~h}$ later.

To initiate these replicates, we separately grew the competitor genotype and the focal genotypes overnight (for $16 \mathrm{~h}$ ) in liquid $\mathrm{KB}$ medium at $28^{\circ} \mathrm{C}$ in an orbital shaker (at 150 r.p.m.). We then introduced $6 \mu \mathrm{l}$ of these overnight samples to each appropriate vial. Additionally, all vials were inoculated with phage, as described above. Replicates were destructively harvested every $24 \mathrm{~h}$ for 10 days to measure bacterial diversity and abundances (see below). We used a total of 360 vials, i.e., 12 treatments $\times 3$ replicates $\times 10$ destructive harvests.

This experimental design allowed us to test if the effect of immigration history on diversification depended on the prior evolutionary history of the focal genotype. Additionally, inclusion of phage in all replicates enabled us to determine if diversification was affected by prior evolution with the exploiters that the focal genotype would encounter in the new habitat.

MEASURING BACTERIAL MORPHOTYPE DIVERSITY AND ABUNDANCES For each harvest (every $24 \mathrm{~h}$ ), we determined cell densities of different morphotypes by counting colonies after 2 days of growth on $\mathrm{KB}$ agar supplemented with $50 \mu \mathrm{g} \mathrm{ml}^{-1}$ 5-bromo-4-chloro3-indolyl-beta-D-galactopyranoside (X-gal). Colony morphotype diversity was measured following the standard methods used in 
previous work (Buckling et al., 2003; Meyer and Kassen, 2007). Niche preference of each morphotype was confirmed by observing growth of each genotype in static medium and establishing whether they mainly colonized the liquid phase (SM), the airliquid interface (WS), or the bottom (FS) of the medium. This measure of diversity is ecologically relevant because colony morphology corresponds to spatial niche use. It should be noted, however, that genetic diversity may have also existed within colony morphotypes, which we did not measure.

Ecological differences among these mutants can be regarded as large as those among species in many systems of animals and plants, for three reasons. First, previous research has identified the genetic bases of the colony morphotypes (Spiers et al., 2002, 2003; Kassen and Rainey, 2004; Spiers and Rainey, 2005), indicating that they reflect genetic variation rather than phenotypic variation within the same genotype. Second, when colonies of a given morphotype were isolated, inoculated into a fresh medium, grown for 2 days, and plated, they produced the same colony morphotype (Fukami et al., 2007), further confirming that the colony morphotypes have genetic bases. Third, colony morphology corresponds to spatial niche differentiation that is of similar or greater ecological magnitude compared to that found among species in many other systems. However, in future research, full genome sequencing of different morphotypes should be helpful in further uncovering the genetic mechanisms underlying diversification in this system.

\section{STATISTICAL ANALYSES}

Data were assessed for normality and log-transformed when necessary prior to analyses. Two-way analysis of variance (ANOVA) was used to test for effects of length of time of prior evolution, prior evolution in the presence of phage, and their interaction on the following response variables: the number of morphotypes observed (time-averaged for days 8-10; Figure 1; Tables 1-3), differences in the number of morphotypes observed (time-averaged

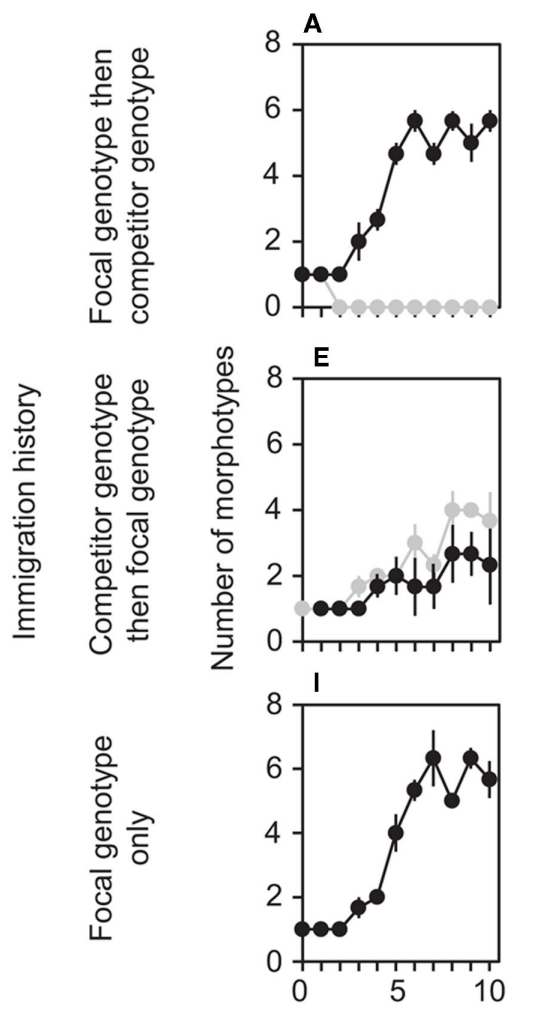

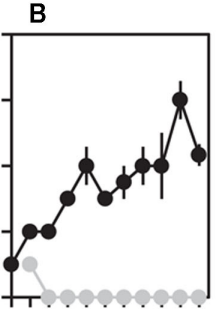
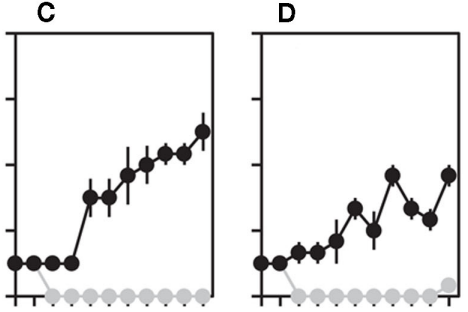

Time **

Phage **

$\mathrm{T} \times \mathrm{P}^{* *}$
F

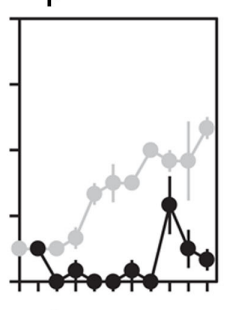

J

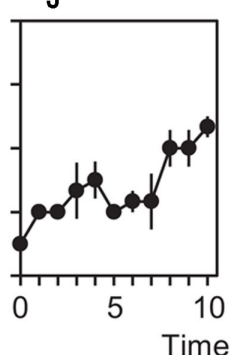

Tim

Time

Adaptation

for 3 days

with phage
Adaptation

for 3 days

without phage
G

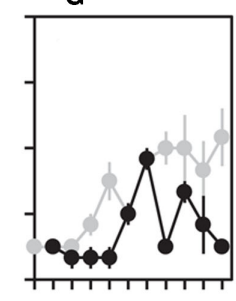

K

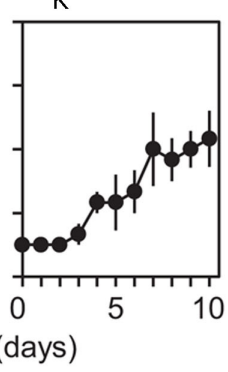

Adaptation for 27 days with phage
H

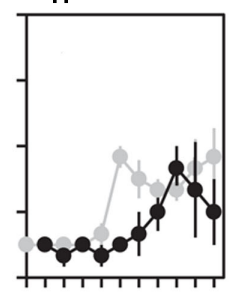

L

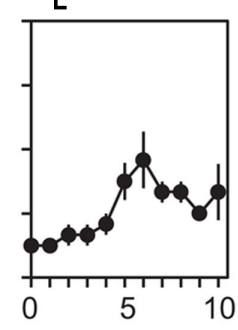

Adaptation

for 27 days

without phage
Time n.s.

Phage n.s.

TxP n.s.

Time **

Phage **

TxP n.s.

Prior evolutionary history
FIGURE 1 | Effects of prior evolutionary history and immigration history on bacterial diversification. Black and gray lines indicate focal and competitor genotypes, respectively. Each panel $(\mathbf{A}-\mathbf{L})$ shows mean number of morphotypes \pm SEM $(n=3)$ for the corresponding treatment of immigration history and prior evolutionary history. For each treatment of immigration history, two-way ANOVA results are indicated as follows: n.s. denotes $p>0.05$, *denotes $p<0.05$, and ${ }^{*}$ denotes $p<0.01$ (see Tables 1-3). "Time," "Phage," and "T $\times P$ " refer to the effect of length of time of prior adaptation, of prior evolution with phage, and of their interaction, respectively. 
for days 8-10) between early immigration and late-immigration treatments (Figure 2; Table 4), and total number of morphotypes descended from both the focal genotype and the competitor genotype (time-averaged for days 0-10; Figure 4; Table 5). All statistical results were for two-tailed tests with $\alpha=0.05$. Statistical analyses were performed using JMP v.8 (SAS Institute Inc.).

\section{RESULTS AND DISCUSSION}

Genotypes differing in prior evolutionary history showed significant differences in the degree to which immigration history affected diversification (Figures 1 and 2). In three of the four prior-history treatments, after approximately 35 bacterial generations (at days 8-10), the focal genotype diversified to a greater extent when it was introduced before, rather than after, its competitor (compare Figures $1 \mathrm{~A}$ vs. 1E, $\mathbf{1 B}$ vs. 1F, and $\mathbf{1 C}$ vs. 1G, see also Figure 2). This result is an expected outcome from previous work (Brockhurst et al., 2007; Fukami et al., 2007). However, when the focal genotype had a long history of adaptation to the laboratory environment in the absence of phage, immigration history did not have a significant effect on the extent of diversification (compare Figures 1D vs. 1H, see also Figure 2).

Why did the importance of immigration history depend on prior evolutionary history? To address this question, we examined the effect of prior evolutionary history within each of the two immigration treatments separately, i.e., when the focal genotype was introduced first and when it was introduced after the competitor. When introduced first, the focal genotype almost completely suppressed the competitor genotype, both in diversity (Figures 1A-D) and abundance (Figures 3A-D), regardless of prior evolutionary history. Therefore, diversification of early immigrants can be considered equivalent to diversification following a single immigration event, simplifying interpretation of results. In fact, when the focal genotype was introduced alone, diversification patterns resembled those observed in the early immigrant treatments (compare Figures 1A-D vs. 1I-L).

We expected that, if introduced alone, genotypes with shorter prior history, and therefore with decreased opportunity for previous adaptation to the novel environment, would diversify more extensively. Two possible mechanisms underlie this expectation. First, both theory (Wright, 1932; Dobzhansky, 1937; Kauffman, 1989; Whitlock et al., 1995) and empirical evidence from P. fluorescens (Buckling et al., 2003) indicate that poorly adapted genotypes have a high potential to diversify via ascending of multiple adaptive peaks. If an immigrant is already adapted to a particular peak, it may be difficult to shift to a new peak, consequently hampering diversification via niche specialization (Buckling et al., 2003; Brockhurst et al., 2007). Second, poorly adapted genotypes may be unable to out-compete descendant genotypes, facilitating the descendants' increase in abundance once they arise by mutation. Although our data do not allow us to ascertain which of these two mechanisms (or both) operated, our results are consistent with the expectation that shorter prior adaptation to the environment results in more extensive diversification (compare Figures 1I vs. $1 \mathrm{~K}$ and $1 \mathrm{~J}$ vs. $1 \mathrm{~L})$.

Table 1 | ANOVA results on the number of morphotypes (time-averaged for days 8-10) for focal genotypes introduced $24 \mathrm{~h}$ before the competitor genotype.

\begin{tabular}{|c|c|c|c|c|c|}
\hline Factor & df & SS & MS & $F$ & $p$ \\
\hline Time & 1 & 2.99 & 2.99 & 20.10 & 0.002 \\
\hline Phage & 1 & 1.80 & 1.80 & 12.10 & 0.008 \\
\hline Time $\times$ phage & 1 & 2.99 & 2.99 & 20.10 & 0.002 \\
\hline Error & 8 & 1.19 & 0.15 & & \\
\hline
\end{tabular}

Table 2 | ANOVA results on the number of morphotypes (time-averaged for days 8-10) for focal genotypes introduced $24 \mathrm{~h}$ after the competitor genotype.

\begin{tabular}{|c|c|c|c|c|c|}
\hline Factor & df & sS & MS & $\boldsymbol{F}$ & $p$ \\
\hline Time & 1 & 0.59 & 0.59 & 0.60 & 0.461 \\
\hline Phage & 1 & 0.04 & 0.04 & 0.04 & 0.847 \\
\hline Time $\times$ phage & 1 & 4.48 & 4.48 & 4.58 & 0.065 \\
\hline Error & 8 & 7.83 & 0.98 & & \\
\hline
\end{tabular}

Table 3 | ANOVA results on the number of morphotypes (time-averaged for days 8-10) for focal genotypes introduced alone.

\begin{tabular}{|c|c|c|c|c|c|}
\hline Factor & df & SS & MS & $\boldsymbol{F}$ & $p$ \\
\hline Time & 1 & 8.91 & 8.91 & 20.02 & 0.002 \\
\hline Phage & 1 & 6.75 & 6.75 & 15.17 & 0.005 \\
\hline Time $\times$ phage & 1 & 1.00 & 0.00 & 0.00 & 1.000 \\
\hline Error & 8 & 3.56 & 0.45 & & \\
\hline
\end{tabular}


We also expected that genotypes that previously evolved in the presence of phage would diversify more than those that did not evolve with phage. There are three possible mechanisms for this expectation. First, previous work has shown that reduction in $P$. fluorescens density by phage results in reduced resource competition, weakening selection pressure for competitive ability (Buckling and Rainey, 2002). Genotypes that previously evolved with phage may therefore be relatively poorly adapted to the laboratory environment, thereby facilitating subsequent diversification as discussed above. Second, genotypes that previously evolved with phage multiplied more quickly (compare Figures 3I vs. 3J and 3K vs. $3 \mathrm{~L}$ ), which may have caused more intense competition, resulting in more extensive diversification. Third, previous work has also found the existence of fitness trade-offs in P. fluorescens between phage resistance and competitive ability (Brockhurst et al., 2004). With such trade-offs, genotypes that previously evolved with phage may be relatively weak in competition and may not strongly suppress descendant mutants, thereby allowing adaptive radiation. Although we cannot determine which mechanism(s) operated, we found that, as expected, prior evolution with phage led to more extensive diversification (compare Figures $1 \mathrm{I}$ vs. 1J and $1 \mathrm{~K}$ vs. 1L).

In contrast to these results for early immigrants, prior evolutionary history did not significantly affect the extent of diversification of late immigrants (Figures 1E-H). Diversification of late immigrants was consistently low regardless of prior evolutionary history (Figures 1E-H). We hypothesize that any potential effect of prior evolutionary history on diversification was overwhelmed by strong niche pre-emption by the early-arriving competitor genotype (Brockhurst et al., 2007; Fukami et al., 2007), which fared better in both diversity (Figures 1E-H) and abundance (Figures 3E-H) by virtue of early arrival. If niche pre-emption was important, diversification should be deterministic when overall diversity of all genotypes descended from both immigrants is considered. Our data are consistent with this expectation, with no significant difference in total diversity between prior-history treatments (Figure 4).
Taken together, our data, combined with previous evidence (Buckling and Rainey, 2002; Buckling et al., 2003; Brockhurst et al., 2004, 2007; Fukami et al., 2007), provide likely explanations for why the importance of immigration history depended on prior evolutionary history, which can be summarized as follows. The focal genotype usually diversified more extensively when it arrived early, due to niche pre-emption (Brockhurst et al., 2007; Fukami et al., 2007). However, this priority effect did not occur if the focal genotype previously had a long period of adaptation in the absence of phage. Genotypes with this history had such a heavily compromised ability to diversify that the extent of diversification after early arrival was indistinguishable from the consistently low level of diversification after late arrival of any immigrant (Figures 1D vs. 1E-H).

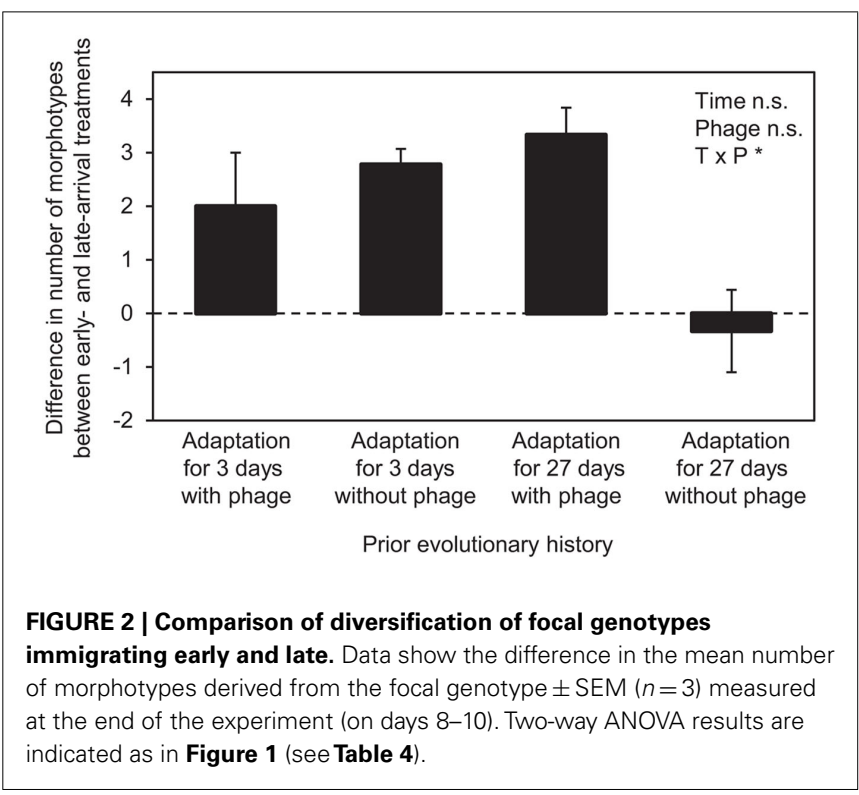

Table 4 | ANOVA results on the number of morphotypes (time-averaged for days 8-10) for the difference between early and late arrival of focal genotypes.

\begin{tabular}{|c|c|c|c|c|c|}
\hline Factor & df & SS & MS & $\boldsymbol{F}$ & $p$ \\
\hline Time & 1 & 7.79 & 7.79 & 4.97 & 0.056 \\
\hline Phage & 1 & 1.56 & 1.56 & 1.00 & 0.347 \\
\hline Time $\times$ phage & 1 & 12.67 & 12.67 & 8.09 & 0.021 \\
\hline Error & 8 & 12.53 & 1.57 & & \\
\hline
\end{tabular}

Table 5 | ANOVA results on the total number of morphotypes from both the competitor genotype and the focal genotype (time-averaged for days 0-10).

\begin{tabular}{|c|c|c|c|c|c|}
\hline Factor & df & SS & MS & $\boldsymbol{F}$ & $p$ \\
\hline Time & 1 & 0.23 & 0.23 & 0.08 & 0.785 \\
\hline Phage & 1 & 1.13 & 1.13 & 0.40 & 0.545 \\
\hline Time $\times$ phage & 1 & 0.76 & 0.76 & 0.27 & 0.617 \\
\hline Error & 8 & 22.38 & 2.80 & & \\
\hline
\end{tabular}




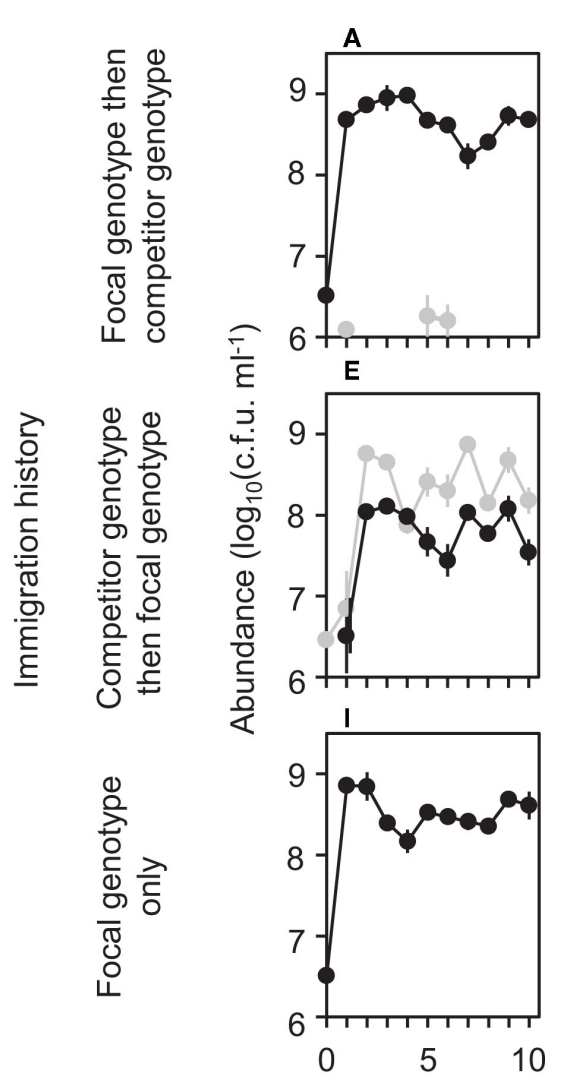

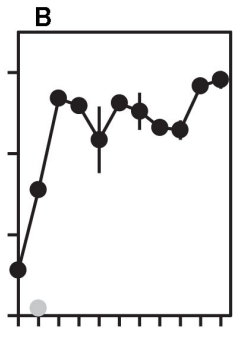

F
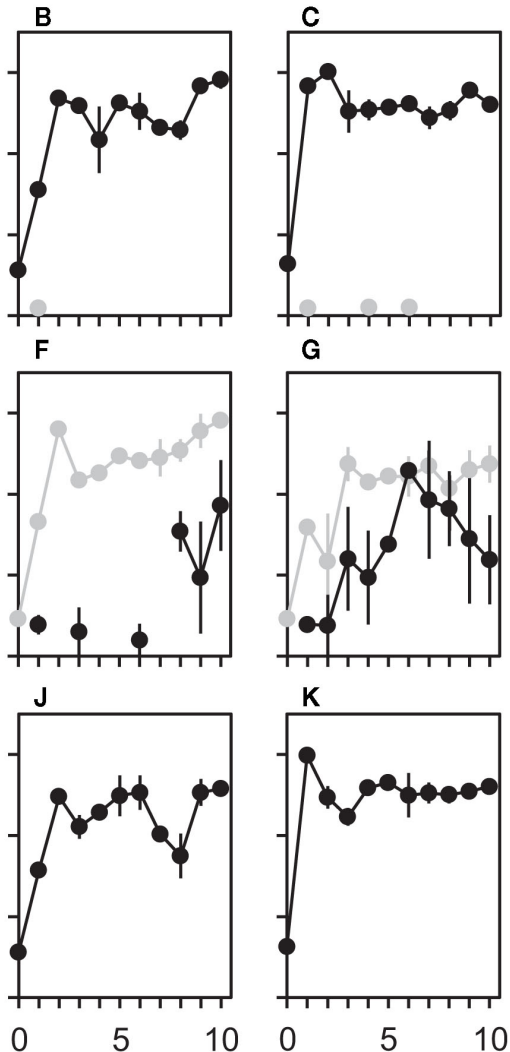

G

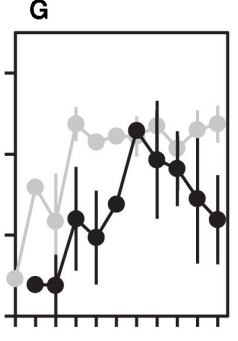

K

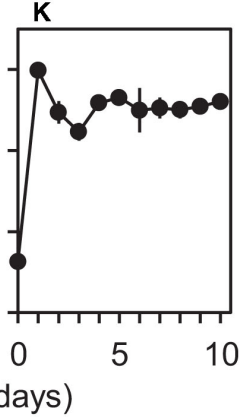

Adaptation

for 3 days

with phage
Adaptation

for 3 days

without phage

\begin{abstract}
Adaptation
for 27 days

with phage
\end{abstract}

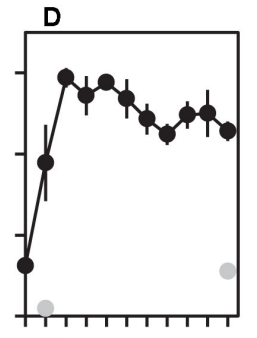

H
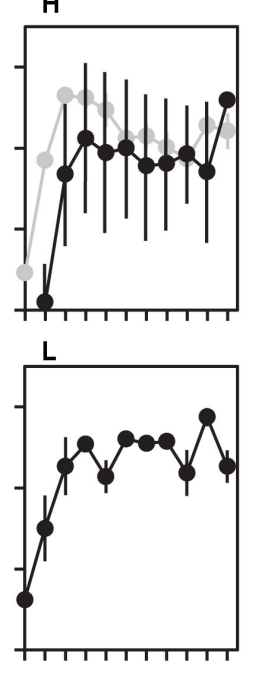

$\begin{array}{lll}0 & 5 & 10\end{array}$

Adaptation

for 27 days

without phage

Prior evolutionary history

FIGURE 3 | Effects of prior evolutionary history and immigration history on bacterial abundance. Black lines indicate focal genotype and gray lines indicate competitor genotype. Each panel $(\mathbf{A}-\mathbf{L})$ shows mean abundance $\left[\log _{10}\left(\mathrm{cfu} \mathrm{ml}^{-1}\right)\right] \pm \mathrm{SEM}(n=3)$ for the corresponding treatment of immigration history and prior evolutionary history.

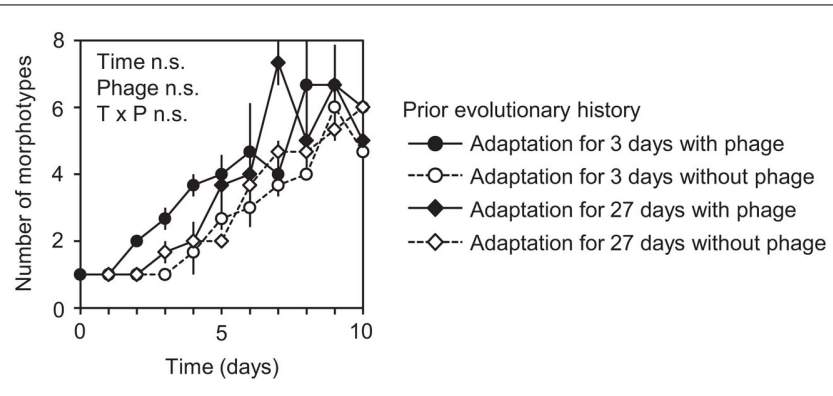

FIGURE 4 | Temporal change in total number of morphotypes after the competitor genotype was introduced on day 0 and the focal genotype on day 1. Data show mean number of morphotypes (those derived from focal and competitor genotypes combined) \pm SEM $(n=3)$. Two-way ANOVA results are indicated as in Figure $\mathbf{1}$ (see Table 5).
The mechanisms indicated in our experiment may also explain some of the variation in the extent of diversification in other systems of micro- and macro-organisms, particularly those involving discrete, replicated habitats such as islands, lakes, and mountaintops. Some of the evolutionary mechanisms that influence bacteria, including lateral gene transfer, uptake of foreign DNA from the environment, mobile DNA elements, recombination machinery, and high mutation rates, may make their diversification different from that of macro-organisms. Nevertheless, evolutionary priority effects similar to those we found in $P$. fluorescens may explain variation in diversification in macro-organisms as well. Possible examples include Macaronesian plants (Silvertown, 2004), African lake cichlids (Seehausen, 2007), and Hawaiian Tetragnatha spiders (Gillespie, 2004). Further, in some systems where the extent of diversification varies considerably, e.g., Caribbean Anolis lizards (Calsbeek and Cox, 2010), mainland source populations appear to have evolved in 
the presence of exploiters (predators), whereas islands where founders immigrated and diversified differ in the occurrence of exploiters, indicating the potential for exploiters to affect diversification.

\section{CONCLUSION}

Historical contingency undermines our ability to explain community assembly because it is difficult to ascertain past events in detail (Gould, 1989; Schluter, 2000; Gavrilets and Losos, 2009; Fukami, 2010). Not all aspects of history are equally complicated, however. In many communities of both micro- and macro-organisms, immigration history is not possible to infer, but adaptation history often is through the combined use of geological, biogeographical, and phylogenetic information (Emerson and Gillespie, 2008; Losos and Ricklefs, 2009; Glor, 2010). Thus, if the hypothesis we propose here is correct, it means that the evolutionary importance of historical events that cannot be reconstructed (i.e., subtle differences in early immigration history) may nevertheless be possible

\section{REFERENCES}

Brockhurst, M. A., Colegrave, N., Hodgson, D. J., and Buckling, A. (2007). Niche occupation limits adaptive radiation in experimental microcosms. PLoS ONE 2, e193-e195. doi:10.1371/journal.pone.0000193

Brockhurst, M. A., Morgan, A. D., Rainey, P. B., and Buckling, A. (2003). Population mixing accelerates coevolution. Ecol. Lett. 6, 975-979.

Brockhurst, M. A., Rainey, P. B., and Buckling, A. (2004). The effect of spatial heterogeneity and parasites on the evolution of host diversity. Proc. Biol. Sci. 271, 107-111.

Buckling, A., and Rainey, P. B. (2002). The role of parasites in sympatric and allopatric host diversification. Nature 420, 496-499.

Buckling, A., Wills, M. A., and Colegrave, N. (2003). Adaptation limits diversification of experimental bacterial populations. Science 302, 2107-2109.

Cadotte, M. W., Drake, J. A., and Fukami, T. (2005). Constructing nature: laboratory models as necessary tools for investigating complex ecological communities. Adv. Ecol. Res. 37, 333-353.

Calsbeek, R., and Cox, R. M. (2010). Experimentally assessing the relative importance of predation and competition as agents of selection. Nature 465, 613-616.

Carpenter, S. R. (1996). Microcosm experiments have limited relevance for community and ecosystem ecology. Ecology 77, 677-680.

Chase, J. M. (2003). Community assembly: when should history matter? Oecologia 136, 489-498.
Dobzhansky, T. (1937). Genetics and the Origin of Species. New York: Columbia University Press.

Emerson, B. C., and Gillespie, R. G. (2008). Phylogenetic analysis of community assembly and structure over space and time. Trends Ecol. Evol. (Amst.) 23, 619-630.

Fukami, T. (2010). "Community assembly dynamics in space," in Community Ecology: Processes, Models, and Applications, eds H. A. Verhoef and P. J. Morin (Oxford: Oxford University Press), 45-54.

Fukami, T., Beaumont, H. J. E., Zhang, X.-X., and Rainey, P. B. (2007). Immigration history controls diversification in experimental adaptive radiation. Nature 446, 436-439.

Gavrilets, S., and Losos, J. B. (2009). Adaptive radiation: contrasting theory with data. Science 323, 732-736.

Gillespie, R. G. (2004). Community assembly through adaptive radiation in Hawaiian spiders. Science 303, 356-359.

Gillespie, R. G., and Emerson, B. C. (2007). Adaptation under a microscope. Nature 446, 386-387.

Glor, R. E. (2010). Phylogenetic insights on adaptive radiation. Annu. Rev. Ecol. Evol. Syst. 41, 251-270.

Gould, S. J. (1989). Wonderful Life: The Burgess Shale and the Nature of History. New York: W. W. Norton Press.

Hairston, N. G. Jr., Ellner, S. P., Geber, M. A., Yoshida, T., and Fox, J. A. (2005). Rapid evolution and the convergence of ecological and evolutionary time. Ecol. Lett. 8, 1114-1127.

Hubbell, S. P. (2001). The Unified Neutral Theory of Biodiversity and

to predict by analyzing more tractable aspects of history (i.e., immigrants' prior adaptation history). For this reason, we believe it will be worthwhile to evaluate the generality and mechanistic bases of our hypothesis in greater detail to gain an improved understanding of immigration, diversification, and community assembly.

\section{ACKNOWLEDGMENTS}

We thank Julia Borden, Benjamin Callahan, Whitney Hoehn, Nathan Kim, Christine Kyauk, Katrina Luna, Sharia Mayfield, and Aaron Wacholder for laboratory assistance. We thank Paul Rainey and Xue-Xian Zhang for providing us with P. fluorescens SBW25 (wild type) and P. fluorescens SBW25 lacZ, and Angus Buckling for providing us with phage $\Phi 2$. We thank Bertus Beaumont, Benjamin Callahan, Sinead Collins, Rodolfo Dirzo, James Estes, Diana Nemergut, Paul Rainey, Uli Stingl, John Thompson, Susannah Tringe, and David Wardle for comments. Financial support was provided by Stanford University.

Biogeography. Princeton: Princeton University Press.

Kassen, R., Llewellyn, M., and Rainey, P. B. (2004). Ecological constraints on diversification in a model adaptive radiation. Nature 451, 984-988.

Kassen, R., and Rainey, P. B. (2004). The ecology and genetics of microbial diversity. Annu. Rev. Microbiol. $58,207-231$.

Kauffman, S. A. (1989). Cambrian explosion and Permian quiescence: implications of rugged fitness landscapes. Evol. Ecol. 3, 274-281.

Losos, J. B., and Ricklefs, R. E. (2009). Adaptation and diversification on islands. Nature 457, 830-836.

Meyer, J. R., and Kassen, R. (2007). The effects of competition and predation on diversification in a model adaptive radiation. Nature 446, 432-435.

Rainey, P. B., and Travisano, M. (1998). Adaptive radiation in a heterogeneous environment. Nature 394 69-72.

Samuels, C. L., and Drake, J. A. (1997) Divergent perspectives on community convergence. Trends Ecol. Evol. (Amst.) 12, 427-432.

Schluter, D. (2000). The Ecology of Adaptive Radiation. Oxford: Oxford University Press.

Schoener, T. W. (2011). The newest synthesis: understanding the interplay of evolutionary and ecological dynamics. Science 331, 426-429.

Seehausen, O. (2007). Chance, historical contingency, and ecological determinism jointly determine the rate of adaptive radiation. Heredity 99, 361-363.
Silvertown, J. (2004). The ghost of competition past in the phylogeny of island endemic plants. J. Ecol. 92, 168-173.

Simpson, G. G. (1953). The Major Features of Evolution. New York: Columbia University Press.

Spiers, A. J., Bohannon, J., Gehrig, S. M., and Rainey, P. B. (2003). Biofilm formation at the air-liquid interface by the Pseudomonas fluorescens SBW25 wrinkly spreader requires an acetylated form of cellulose. Mol. Microbiol. 50, 15-27.

Spiers, A. J., Kahn, S. G., Bohannon, J., Travisano, M., and Rainey, P. B. (2002). Adaptive divergence in experimental populations of Pseudomonas fluorescens. I. Genetic and phenotypic bases of wrinkly spreader fitness. Genetics 161, 33-46.

Spiers, A. J., and Rainey, P. B. (2005). The Pseudomonas fluorescens SBW25 wrinkly spreader A-L biofilm requires attachment factor, cellulose fibre and LPS interactions to maintain strength and integrity. Microbiology 151, 2829-2839.

Urban, M. C., and De Meester, L. (2009). Community monopolization: local adaptation enhances priority effects in an evolving metacommunity. Proc. Biol. Sci. 276, 4129-4138.

Whitlock, M. C., Phillips, P. C., Moore, F. B. G., and Tonsor, S. (1995). Epistasis and multiple fitness peaks. Annu. Rev. Ecol. Evol. Syst. 26, 601-629.

Wright, S. (1932). "The roles of mutation, inbreeding, crossbreeding and selection in evolution," in Proceedings 6th International Congress of Genetics, Ithaca, NY, 356-366. 
Zhang, X.-X., and Rainey, P. B. (2007). Construction and validation of a neutrally-marked strain of Pseudomonas fluorescens SBW25. J. Microbiol. Methods 71, 78-81.

Conflict of Interest Statement: The authors declare that the research was conducted in the absence of any commercial or financial relationships that could be construed as a potential conflict of interest.

Received: 21 September 2011; accepted: 21 December 2011; published online: 09 January 2012.
Citation: Knope ML, Forde SE and Fukami T (2012) Evolutionary history, immigration history, and the extent of diversification in community assembly. Front. Microbio. 2:273. doi: 10.3389/fmicb.2011.00273

This article was submitted to Frontiers in Evolutionary and Genomic Microbiology, a specialty of Frontiers in Microbiology.
Copyright (c) 2012 Knope, Forde and Fukami. This is an open-access article distributed under the terms of the Creative Commons Attribution Non Commercial License, which permits non-commercial use, distribution, and reproduction in other forums, provided the original authors and source are credited. 